\title{
Usefulness of biopsy by office hysteroscopy for endometrial cancer: A case report
}

\author{
KENBUN SONE, SATOKO EGUCHI, KAYO ASADA, FUTABA INOUE, YUICHIRO MIYAMOTO, \\ MICHIHIRO TANIKAWA, TETSUSHI TSURUGA, MAYUYO MORI-UCHINO, YOKO MATSUMOTO, \\ OSAMU HIRAIKE-WADA, KATSUTOSHI ODA, YUTAKA OSUGA and TOMOYUKI FUJII
}

\begin{abstract}
Department of Obstetrics and Gynecology, Graduate School of Medicine, The University of Tokyo, Tokyo 113-8655, Japan
\end{abstract}
Received June 30, 2019; Accepted March 26, 2020

DOI: $10.3892 / \mathrm{mco} .2020 .2053$

\begin{abstract}
A diagnostic biopsy for endometrial cancer is performed via dilation and curettage (D\&C). However, D\&C may miss endometrial cancer lesions due to of its 'blind' approach. Hysteroscopy is a useful method that can be used to detect endometrial cancer lesions. In addition, office hysteroscopy is easy to be scheduled and does not require anesthesia. The patient was a 40-year-old woman with suspected endometrial cancer; however, it could not be diagnosed by D\&C and biopsy using hysteroscopy during hospitalization. Office hysteroscopy during the proliferative phase indicated that the suspicious endometrial cancerous lesion was minimal at the isthmus of the uterus with atypical vessels and a white spot, for which biopsy was performed. Pathological diagnosis was endometrioid carcinoma with squamous differentiation, G1. Therefore, total laparoscopic hysterectomy, bilateral salpingo-oophorectomy and pelvic lymphadenectomy were performed. In this case, it was difficult to detect minimal lesion in the secretory phase because the endometrial thickness hid the endometrial cancer. It is easy to perform office hysteroscopy in the proliferative phase. This case indicated that office hysteroscopy is a useful method to diagnose and perform biopsy for minimal lesions.
\end{abstract}

\section{Introduction}

Endometrial cancer is one of the most common gynecological cancers and its incidence has increased worldwide in recent years (1). Although low-risk and early stage endometrial

Correspondence to: Dr Kenbun Sone, Department of Obstetrics and Gynecology, Graduate School of Medicine, The University of Tokyo, 7-3-1 Hongo, Bunkyo-ku, Tokyo 113-8655, Japan

E-mail: ksone5274@gmail.com

Abbreviations: D\&C, dilation and curettage; MRI, magnetic resonance imaging

Key words: office hysteroscopy, endometrial cancer, biopsy, dilation and curettage, proliferative phase cancer patients have a favorable prognosis, there are few effective chemotherapy options for high-risk patients. Although Pembrolizumab (a PD-1 inhibitor) was approved for use in patients with microsatellite instability-high tumors, which are often seen in endometrial cancer, no molecular targeted therapies for endometrial cancer have been approved to date $(2,3)$. Thus, it is important to diagnose endometrial cancer at an early stage. Dilation and curettage (D\&C) is a common diagnostic procedure. Although almost all institutions perform $\mathrm{D} \& \mathrm{C}$ for examination of endometrial cancer (4), there is a weakness about using D\&C for the diagnosis because this blind procedure might miss endometrial cancer (5). Therefore, this procedure has a high rate of false negatives. For example, Liu et al showed that failure rates with D\&C were $7.75 \%$ (6). In contrast, hysteroscopy is generally the common standard procedure for examining endometrial lesions, evaluating the uterine cavity directly, and performing the biopsy. In addition, office hysteroscopy is a minimally invasive procedure and easier to schedule than hysteroscopic biopsy in hospitalization. Recently, some reports indicated that hysteroscopy is a useful procedure for diagnosing endometrial cancer (3)

Here, we report the case of a patient with suspected endometrial cancer; however, it could not be diagnosed via D\&C and hysteroscopic biopsy during hospitalization, and she was diagnosed with the condition via office hysteroscopic biopsy.

\section{Case report}

The patient was a 40-year-old woman with no history of pregnancy or delivery. Her menstruation is irregular. She visited the local clinic for hypermenorrhea. Endometrial polyp was detected by transvaginal echo and transcervical resection was performed. Pathological finding was atypical glands of uterine corpus. Atypical glands might suggest atypical endometrioid hyperplasia complex or endometrioid adenocarcinoma (Fig. 1A). It is necessary to rule out atypical polypoid adenomyoma. She visited our hospital for further examination. During gynecological examination, abnormal findings were not detected. Endometrial thickness was determined via ultrasound. Blood test, cytological and histological examination did not reveal 
A

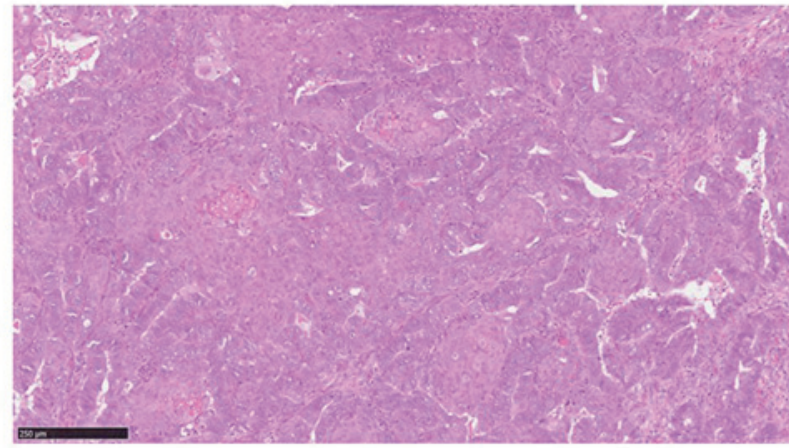

B

C
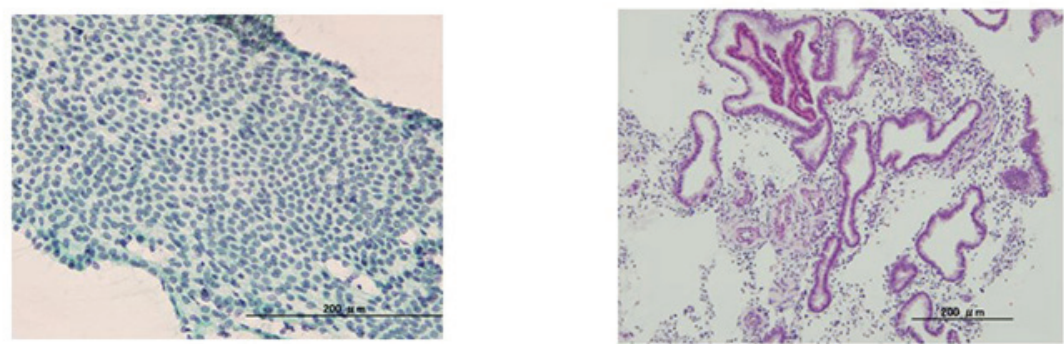

Figure 1. Microscopic findings before D\&C. (A) Histological findings of the polyp biopsied at the University of Tokyo Hospital, (B) Cytological findings of the endometrium at the University of Tokyo Hospital and (C) Histological findings of the endometrium at the University of Tokyo Hospital. D\&C, dilation and curettage.

A

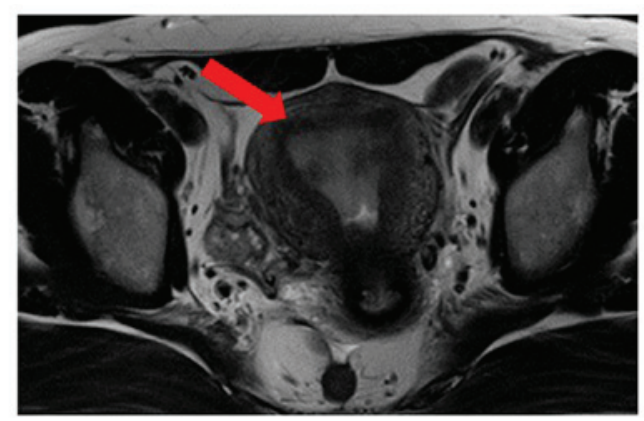

C

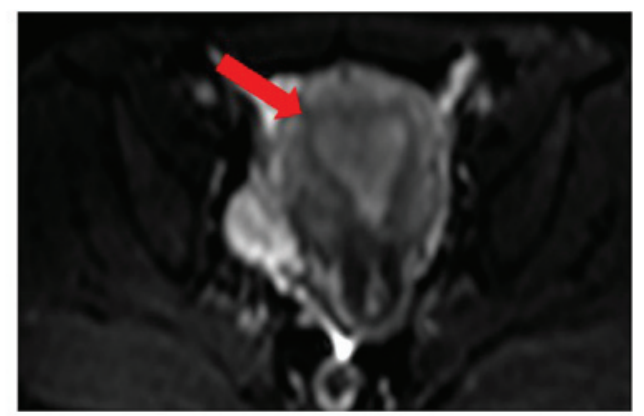

B

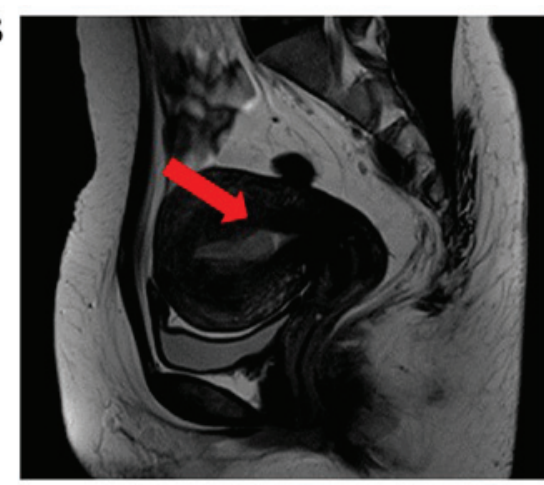

Figure 2. MRI findings. (A) Axial T2-weighted image (T2W1), (B) Sagittal T2-weighted image (T2W1). Although (C) Diffusion weighted imaging MRI showed endometrial thickness (red arrows) and endometrial cancer was not suspected. MRI, magnetic resonance imaging.

abnormal findings (Fig. 1B and C). Additionally,pathological examination was not abnormal in our hospital. Magnetic resonance imaging (MRI) was performed in our hospital, and it showed endometrial thickening $(10 \mathrm{~mm})$ (Fig. 2). There was no evidence of invasion to the wall of the uterus. On computed tomography scan, there were no metastatic lesions or enlarged lymph nodes. Therefore, we performed hysteroscopic biopsy and D\&C under anesthesia after admission. However, there were no hysteroscopic findings, and pathological findings showed the endometrium to be in secretory phase without malignancy (Fig. 3). Although we followed up the patient to perform cytological examination of the endometrium in outpatient clinic after the operation, we did not detect abnormal findings. We planned to perform 
A

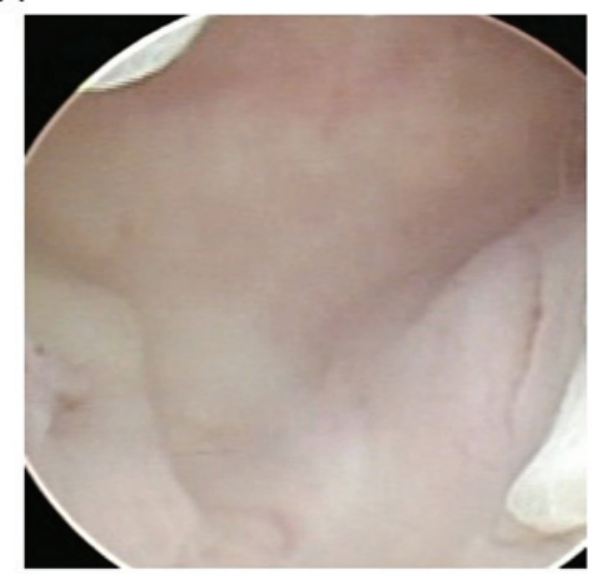

B

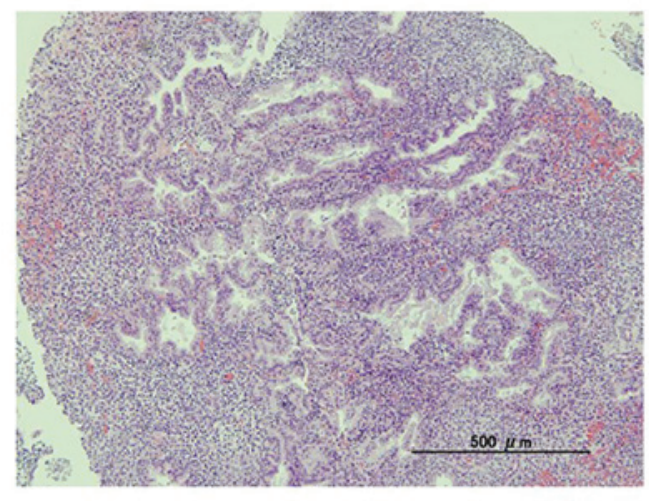

Figure 3. Hysteroscopic findings of the uterine cavity. (A) There was no apparent malignant endometrial lesion in hysteroscopy during hospitalization. The small endometrial polyp was detected using hysteroscopy. (B) Histological findings of the polyp biopsied by hysteroscopy during hospitalization.

A

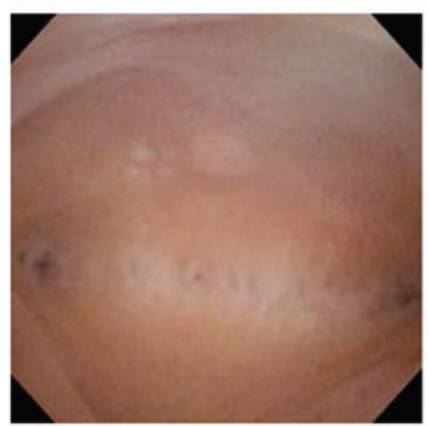

C

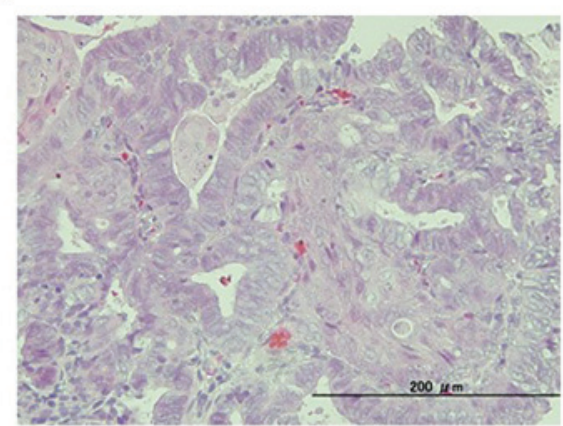

B

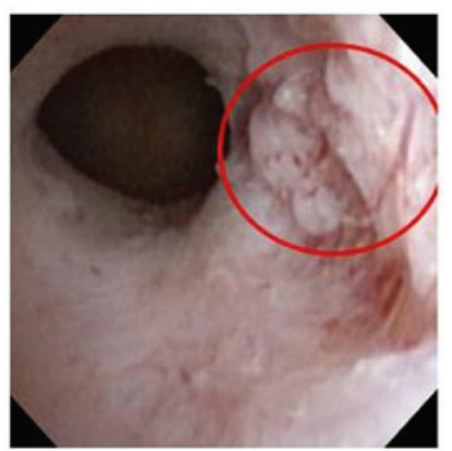

Figure 4. Office hysteroscopic findings of the uterine cavity. (A) No malignant endometrial lesions were indicated at the fundus of uterus. (B) Office hysteroscopy showed that the lesion was small at the isthmus of the uterus with atypical vessels and white spots. (C) Histological findings of the suspected endometrial cancer lesion biopsied by office hysteroscopy. Red circle, lesion.

hysteroscopic biopsy in the outpatient clinic 5 months after the first hysteroscopy since a previous transcervical resection biopsy performed at another hospital found atypical glands in the uterine corpus, which are suggestive of complex atypical endometrial hyperplasia or endometrioid adenocarcinoma. We scheduled the patient to come to our hospital in the proliferative phase. We used TROPHYscope ${ }^{\circledR}$ CAMPO (KARL STORZ) as a hysteroscope. At the start of the examination, the primary access into the uterine cavity was performed with an outer diameter of $2.9 \mathrm{~mm}$ with the corresponding sheath in the passive position. The hysteroscope was introduced into the uterine cavity through this sheath. For endometrial biopsy, an extra operating sheath with a working channel for 5 Fr. instruments was used (7). Office hysteroscopy showed that the suspicious endometrial cancerous lesion was minimal at the isthmus of the uterus with atypical vessels and a white spot, for which biopsy was performed twice (Fig. 4A and B). The pathological finding was endometrioid adenocarcinoma, G1 (Fig. 4C). Our department usually performs pelvic lymphadenectomy in low-risk patients for precise staging according to the guidelines of the Japan Society of Gynecologic Oncology. Therefore, total laparoscopic hysterectomy, bilateral salpingo-oophorectomy, and pelvic lymphadenectomy were 
A

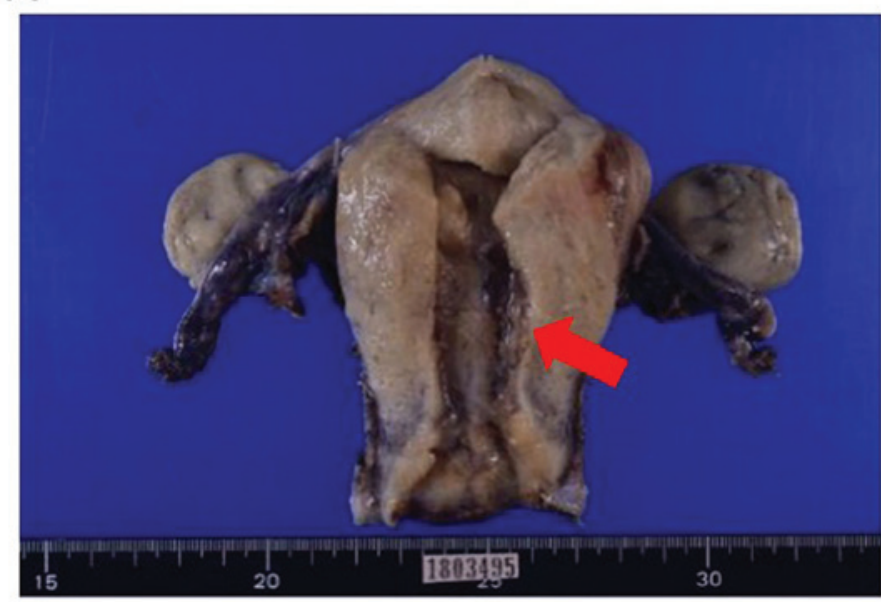

B

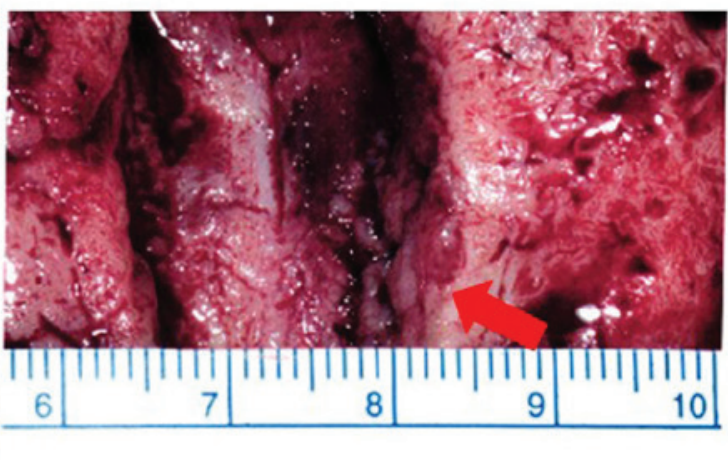

Figure 5. Macroscopic findings. (A) Macroscopic findings suggested the presence of small endometrial cancer at the isthmus of the uterus. (B) Zoomed in view of macroscopic findings. Red arrow, cancerous lesion.

performed. Pathological diagnosis was endometrioid carcinoma with squamous differentiation, G1, 9x7 x6 mm, pT1a, ly(-), v(-), LN(0/39) (Fig. 5). The patient is making steady progress two years after surgery.

\section{Discussion}

We report the case of a 40-year-old woman with suspected endometrial cancer; however, it could not be diagnosed by $\mathrm{D} \& \mathrm{C}$ and biopsy using hysteroscopy in hospitalization. The patient was diagnosed with endometrial cancer via office hysteroscopic biopsy. Increasing tumor size is one of the reasons that enable office hysteroscopy to detect endometrial cancer. In this case, we believe that small polyps suggestive of atypical hyperplasia and endometrial cancer that was revealed in the proliferative phase were the main reason for outpatient hysteroscopy. Lately, the number of patients with endometrial cancer is increasing in developed countries. There are a few methods for the treatment of advanced endometrial cancer (8). It is necessary to diagnose endometrial cancer early to ensure good prognosis. Although endometrial cancer with tumor formation can be easily diagnosed, it is difficult to diagnose it when it is in the early stages. Therefore, the negative predictive value of normal endometrial biopsy is low at $51 \%$. Approximately $3 \%$ of endometrial cancer is missed because of blinded D\&C (9). Some gynecologists used several types of hysteroscopic features of endometrial lesions for diagnosing malignancy, including irregularity of endometrial glands, polypoid pattern, uneven surface, and abnormal endometrial vessels (5). Additionally, some reports suggested that normal hysteroscopic findings do not prove the absence of endometrial lesion. It is necessary to perform endometrial biopsy when there is increased endometrial thickness, with abnormal bleeding (5). The use of blind sampling of endometrium, such as is done via D\&C, is not an accurate method for diagnosing endometrial cancer. We surely can biopsy the malignant endometrial lesion with the use of hysteroscopy with directed biopsy (7). However, we did not detect endometrial lesion in the first hysteroscopy because of endometrial thickness in the secretory phase. Therefore, we performed office hysteroscopy and succeeded in biopsy of malignant endometrial lesion. One of the important advantages of office hysteroscopy is that this procedure does not need hospitalization and anesthesia. The patients can avoid from discomfort of going into the operating room. Therefore, this procedure is considered as minimally invasive and highly tolerable by the patients while still sufficiently accurate for diagnostic purposes. These factors explain why patients tend to prefer the diagnosis of endometrial cancer by office hysteroscopy. Another important point is that it is easier to schedule office hysteroscopy than hysteroscopy during hospitalization. In high volume hospitals such as ours, it is difficult to schedule surgical procedures when compared to a local hospital. We consider the ease of examination an important advantage of office hysteroscopy. In this case, it was difficult to detect minimal lesion in the secretory phase because the endometrial thickness hid the endometrial cancer. It is easy to schedule office surgery in the proliferative phase even if her menstruation is irregular. There are issues with diagnosing endometrial cancer using hysteroscopy. A previous report indicated that hysteroscopy could increase the probability of positive peritoneal cytology (10). However, hysteroscopy does not induce the risk of ovarian or abdominal metastasis. This result suggested that the iatrogenic peritoneal spillage cannot induce metastasis $(11,12)$. In addition, there is the possibility that intracavitary pressure by hysteroscopic fluid induces iatrogenic peritoneal spillage of endometrial cells. No spillage has been reported to occur at pressures $<70 \mathrm{mmHg}$ (13). Another examination method for endometrial cancer is transvaginal ultrasonography. Berretta et al suggested that transvaginal ultrasonography is a useful method for assessing myometrial invasion by endometrial cancer (14). Other reports suggested that tumor size is a useful marker for the surgical staging of endometrial cancer (15).

Taken together, we suggest that office hysteroscopy with directed biopsy is useful for the diagnosis of minimal endometrial cancer. 


\section{Acknowledgements}

No applicable.

\section{Funding}

No funding was received.

\section{Availability of data and materials}

The datasets used and/or analyzed during the current study are available from the corresponding author on reasonable request.

\section{Authors' contributions}

$\mathrm{KS}$ prepared the manuscript and figures. KS, SE, KA, KO, $\mathrm{YO}, \mathrm{TF}$ and $\mathrm{OHW}$ provided medical care for the patients and collected the data. KS, YMi and OHW performed the operation. KS, SE, KA, FI, YMi, MT, TT and MMU participated in the conception and design of the case report. YMa, OHW, KO, YO, and TF revised the manuscript for intellectual content. FI also retrieved the pathology images. KO, YO, and TF supervised the entire project. All authors read and approved the final manuscript.

\section{Ethics approval and consent to participate}

Written informed consent was obtained from the patient in this case. The study design was approved by the Ethics Committee at The University of Tokyo.

\section{Patient consent for publication}

The patient provided written informed consent for the publication of any associated data and accompanying images.

\section{Competing interests}

The authors declare that they have no competing interests.

\section{References}

1. Anderson AS, Key TJ, Norat T, Scoccianti C, Cecchini M, Berrino F, Boutron-Ruault MC, Espina C, Leitzmann M, Powers H, et al: European code against cancer 4th edition: Obesity, body fatness and cancer. Cancer Epidemiol 39: S34-S45, 2014.

2. Lachance JA, Darus CJ and Rice LW: Surgical management and postoperative treatment of endometrial carcinoma. Rev Obstet Gynecol 1: 97-105, 2008.

3. Ott PA, Bang YJ, Berton-Rigaud D, Elez E, Pishvaian MJ, Rugo HS, Puzanov I, Mehnert JM, Aung KL, Lopez J, et al: Safety and antitumor activity of pembrolizumab in advanced programmed death ligand 1-positive endometrial cancer: Results from the KEYNOTE- 028 Study. J Clin Oncol 35: 2535-2541, 2017.
4. Yang B, Xu Y, Zhu Q, Xie L, Shan W, Ning C, Xie B, Shi Y, Luo X, Zhang $\mathrm{H}$ and Chen X: Treatment efficiency of comprehensive hysteroscopic evaluation and lesion resection combined with progestin therapy in young women with endometrial atypical hyperplasia and endometrial cancer. Gynecol Oncol 153: 55-62, 2019.

5. Trojano G, Damiani GR, Casavola VC, Loiacono R, Malvasi A, Pellegrino A, Siciliano V, Cicinelli E, Salerno MG and Battini L: The role of hysteroscopy in evaluating postmenopausal asymptomatic women with thickened endometrium. Gynecol Minim Invasive Ther 7: 6-9, 2018.

6. Liu H, Wang FL, Zhao YM, Yao YQ and Li YL: Comparison of Pipelle sampler with conventional dilatation and curettage (D\&C) for Chinese endometrial biopsy. J Obstet Gynaecol 35: 508-511, 2015.

7. De Wilde RL: Office Hysteroscopy: TROPHYscope CAMPO compact hysteroscope ${ }^{\circledR}$ : Manufacturer: KARL STORZ, Tuttlingen, Germany. J Obstet Gynaecol India 64: 301-303, 2014.

8. Thigpen JT, Brady MF, Homesley HD, Malfetano J, DuBeshter B, Burger RA and Liao S: Phase III trial of doxorubicin with or without cisplatin in advanced endometrial carcinoma: A gynecologic oncology group study. J Clin Oncol 22: 3902-3908, 2004.

9. Yen CF, Chou HH, Wu HM, Lee CL and Chang TC: Effectiveness and appropriateness in the application of office hysteroscopy. J Formos Med Assoc 118: 1480-1487, 2019.

10. Dovnik A, Crnobrnja B, Zegura B, Takac I and Pakiz M Incidence of positive peritoneal cytology in patients with endometrial carcinoma after hysteroscopy vs. dilatation and curettage. Radiol Oncol 51: 88-93, 2017.

11. Ben-Arie A, Tamir S, Dubnik S, Gemer O, Ben Shushan A, Dgani R, Peer G, Barnett-Griness O and Lavie O: Does hysteroscopy affect prognosis in apparent early-stage endometrial cancer? Int J Gynecol Cancer 18: 813-819, 2008.

12. Chen J, Clark LH, Kong WM, Yan Z, Han C, Zhao H, Liu TT, Zhang TQ, Song D, Jiao SM and Zhou C: Does hysteroscopy worsen prognosis in women with type II endometrial carcinoma? PLoS One 12: e0174226, 2017.

13. Baker VL and Adamson GD: Threshold intrauterine perfusion pressures for intraperitoneal spill during hydrotubation and correlation with tubal adhesive disease. Fertil Steril 64: 1066-1069, 1995.

14. Berretta R, Merisio C, Piantelli G, Rolla M, Giordano G, Melpignano $M$ and Nardelli GB: Preoperative transvaginal ultrasonography and intraoperative gross examination for assessing myometrial invasion by endometrial cancer. J Ultrasound Med 27: 349-355, 2008

15. Berretta R, Patrelli TS, Migliavacca C, Rolla M, Franchi L, Monica M, Modena AB and Gizzo S: Assessment of tumor size as a useful marker for the surgical stagingof endometrial cancer. Oncol Rep 31: 2407-2412, 2014.

This work is licensed under a Creative Commons Attribution-NonCommercial-NoDerivatives 4.0 International (CC BY-NC-ND 4.0) License. 